\title{
The Melatonin Receptor Agonist Is Effective for Free-Running Type Circadian Rhythm Sleep Disorder: Case Report on Two Sighted Patients
}

\author{
Mariko Yanagihara, ${ }^{1,2,3}$ Masaki Nakamura, ${ }^{1,2,4}$ Akira Usui, ${ }^{1,2,4}$ Shingo Nishida, ${ }^{1,2,4}$ \\ Eiki Ito, ${ }^{1,2,4}$ Masako Okawa ${ }^{1,2,4,5}$ and Yuichi Inoue ${ }^{1,2,4}$
}

\author{
${ }^{1}$ Japan Somnology Center, Neuropsychiatric Research Institute, Tokyo, Japan \\ ${ }^{2}$ Yoyogi Sleep Disorder Center, Tokyo, Japan \\ ${ }^{3}$ First Department of Internal Medicine, Tokyo Medical University, Tokyo, Japan \\ ${ }^{4}$ Department of Somnology, Tokyo Medical University, Tokyo, Japan \\ ${ }^{5}$ Japan Foundation for Neuroscience and Mental Health, Kodaira, Tokyo, Japan
}

\begin{abstract}
Along with urbanization of the living environment, the number of patients with circadian rhythm sleep disorder (CRSD) has been increasing. There are several treatment candidates for CRSD, such as light therapy, drugs (melatonin and vitamin B12), and sleep hygiene education. However, successful treatment method has not been established. In free-running type (FRT) CRSD, the endogenous circadian rhythm cannot be entrained to the 24-h light-dark cycle, resulting in free running on a cycle $0.5-2.5 \mathrm{~h}$ longer than the 24-h period. This condition is relatively common in blind individuals and is unusual in sighted individuals. Here we report two sighted patients with FRT, successfully treated with a melatonin receptor agonist, ramelteon. Patient 1 (36-year-old female) had suffered from FRT for nearly 4 months after resigning her job. She was given sleep hygiene education together with ramelteon at first and the freerunning cycle stopped after treatment day 15. Triazolam was added from the day 25 to promote earlier sleep onset. And the sleep-wake schedule was normalized by the day 34 . Patient 2 (33-year-old male) had suffered from FRT for nearly 8 months after starting to take a leave of absence from his job. He was given sleep hygiene education and was treated with ramelteon and methylcobalamin. His sleep-wake schedule was normalized from the first treatment day. By the combined treatment with ramelteon, both patients have maintained favorable sleep-wake schedules. The agonist action of ramelteon at the melatonin 2 receptor may have primarily contributed to the cessation of the free-running cycle in these patients.
\end{abstract}

Keywords: circadian rhythm sleep disorder; free running type; melatonin agonist; non-24-hour sleep wake disorder; sighted patient

Tohoku J. Exp. Med., 2014 October, 234 (2), 123-128. C 2014 Tohoku University Medical Press

\section{Introduction}

Along with urbanization of the living environment, the number of patients with circadian rhythm sleep disorder (CRSD) has been increasing. In this condition, sleep disturbance often causes clinically significant distress or impairment of social or occupational functioning. There are several treatment candidates for CRSD such as light therapy, drugs (melatonin and vitamin B12) and sleep hygiene education. However, successful treatment method has not been established. Among these treatments, melatonin or its agonists have been awarded for their fundamental effect on CRSD.

In this paper, we would like to emphasize treatment effects of a melatonin agonist for free-running type (FRT) CRSD. According to the criteria in the International Classification of Sleep Disorders, Second Edition (ICSD-2) (American Academy of Sleep Medicine 2005), FRT is characterized by the condition that the endogenous circadian rhythm cannot be entrained to the 24-h light-dark cycle and instead free-runs on a cycle $0.5-2.5 \mathrm{~h}$ longer than the $24-\mathrm{h}$ period (Hayakawa et al. 2005; Okawa and Uchiyama 2007), and termed "non-24-hour sleep wake disorder" in the ICSD-3 (American Academy of Sleep Medicine 2014). Patients have difficulty in awakening at a scheduled time, general malaise, daytime sleepiness, dysthymia, and severe autonomic nervous system symptoms, all of which come from internal desynchronization of various physiological

Received April 15, 2014; revised and accepted September 5, 2014. Published online September 23, 2014; doi: 10.1620/tjem.234.123. Correspondence: Yuichi Inoue, M.D., Ph.D., Japan Somnology Center, Neuropsychiatric Research Institute, 1-24-10 Yoyogi, Shibuyaku, Tokyo 151-0053, Japan.

e-mail: inoue@somnology.com 
phenomena that follow regular circadian cycles. Unfortunately, since this disorder is not widely recognized, patients are likely to receive inappropriate treatment (Hayakawa et al. 2005).

This condition is relatively common in blind individuals (about $50 \%$ of whom have FRT) (Sack et al. 2000), but is unusual in sighted individuals (Hashimoto et al. 1997; Hayakawa et al. 2005; American Academy of Sleep Medicine 2005, 2014). In blind patients with FRT, the main cause of the disorder is a lack of photic stimulation from the retina to the suprachiasmatic nucleus ( $\mathrm{SCN}$ ), leading to a poorly regulated rhythm of melatonin secretion from the pineal gland (Morgenthaler et al. 2007). In sighted patients, environmental conditions such as decreased or inappropriately timed exposure to light (American Academy of Sleep Medicine 2005), weakening of social zeitgebers such as going to school or a job on time (Okawa and Uchiyama 2007), and impaired response of the circadian clock to the entraining effect of light have been speculated to contribute to the development of FRT. There is other condition of CRSD termed "delayed sleep phase type (DSPT)". Patients with DSPT have difficulty in sleeping and waking up at desirable time and their sleep-wake schedule is fixed at a later time against their will, keeping a cycle of 24 hours and not showing free running. The FRT and DSPT may share common pathophysiology, and in fact, some patients progress from DSPT to FRT (Hayakawa et al. 2005; Okawa and Uchiyama 2007). Abnormal sensitivity to light-induced melatonin suppression in DSPT patients has been reported in previous studies (McArthur et al. 1996; Hashimoto et al. 1997; Aoki et al. 2001). With regard to FRT, a complete mismatch between the serum melatonin rhythm and the sleep-wake rhythm has been reported to exist in some cases (Honma et al. 1997), possibly suggesting that the phase response to melatonin may be impaired in the disorder.

Previously, some patients with FRT have been successfully treated with light therapy (Hayakawa et al. 1998; Okawa et al. 1998b), vitamin B12 (Hoban et al. 1989; Okawa et al. 1990; Ohta et al. 1991; Oren et al. 1997; Watanabe et al. 2000), and melatonin preparations, either separately or in combination (Morgenthaler et al. 2007), but the treatment efficacies of these approaches have not been conclusive. A melatonin receptor agonist, ramelteon, has a longer half-life than melatonin preparations, along with a 6-fold higher affinity for the melatonin (MT) 1 receptor and a 3-fold higher affinity for the MT2 receptor in the SCN (Kato et al. 2005; Karim et al. 2006). It has been widely accepted that agonist action at MT1 provides a sleep-inducing effect and that at MT2 provides a circadian phase-shifting property (Reppert et al. 1995; Richardson et al. 2008). However, the effects of ramelteon treatment for FRT have not yet been determined.

Here, we report two cases of sighted FRT patients who responded to treatment with ramelteon, and briefly discuss the possible mechanisms of action of the melatonin agonist in this disorder.
Each patient provided written informed consent for this study.

\section{Case Presentation}

\section{Patient 1}

A 36-year-old female presented with a chief complaint of sleep-onset insomnia, difficulty awakening in the morning, and intermittent excessive daytime sleepiness. There was no past history of physical disease or sight impairment. Since childhood, she tended to have difficulty falling asleep at night and awakening on schedule in the morning. From around 13 years of age, her sleep onset time was delayed to about 02:00. After beginning to work as a nurse at age 21, she began to suffer from sleep-onset insomnia, physical disturbances, including headaches and dizziness, and depressed mood. From the age of 26, she had repeated bouts of absence, and she resigned from several workplaces. Various kinds of antidepressants and hypnotics were prescribed by her attending physicians for depressed mood and sleep-onset insomnia, but no remarkable improvement of the symptoms was obtained. In January 2011 , at the age of 35, she resigned from her job. Shortly thereafter, her depressed mood decreased but persisted, and sleep-onset insomnia and difficulty awakening in the morning also persisted. In the autumn of that year, both her sleep onset and offset times started to gradually delay by 1-3 h each day. At that time, she stayed in her apartment all day long, going out only a few times per month during the daytime. She came to our sleep disorder clinic seeking treatment for this condition in February 2012. Before then, her sleep onset and offset times had continued to free-run for nearly 4 months, in spite of her efforts to keep to a regular sleep schedule. Based on a thorough clinical interview and her sleep diary, she was diagnosed as having FRT with the absence of any other sleep disorders or primary psychiatric disorders.

We gave her sleep hygiene education, including the instruction to open the curtains of her bedroom as early as possible in the morning, to refrain from watching television and using computers after 21:00, and to turn the lights down in her room at 22:00. However, her sleep onset and offset times continued to free-run after receiving the education. When her sleep onset time came around 21:00, we instructed her to start taking $8 \mathrm{mg}$ of ramelteon at 20:00. Fig. 1 shows the sleep diary after starting this treatment. She initially went to bed at 22:00, but had difficulty falling asleep. During the 10 days from the start of the treatment, her sleep onset time gradually became more and more delayed, just as before the start of treatment. The free running of her sleep phase subsequently diminished until day 15, when sleep onset settled around 02:00 and awakening around 09:00, and then the subjective physical problems almost disappeared. Triazolam $0.25 \mathrm{mg}$ at 23:00 was added from day 25 in order to promote earlier sleep onset. Shortly thereafter, sleep latency was shortened, but this had no effect on either difficulty awakening or morning sleepiness. 


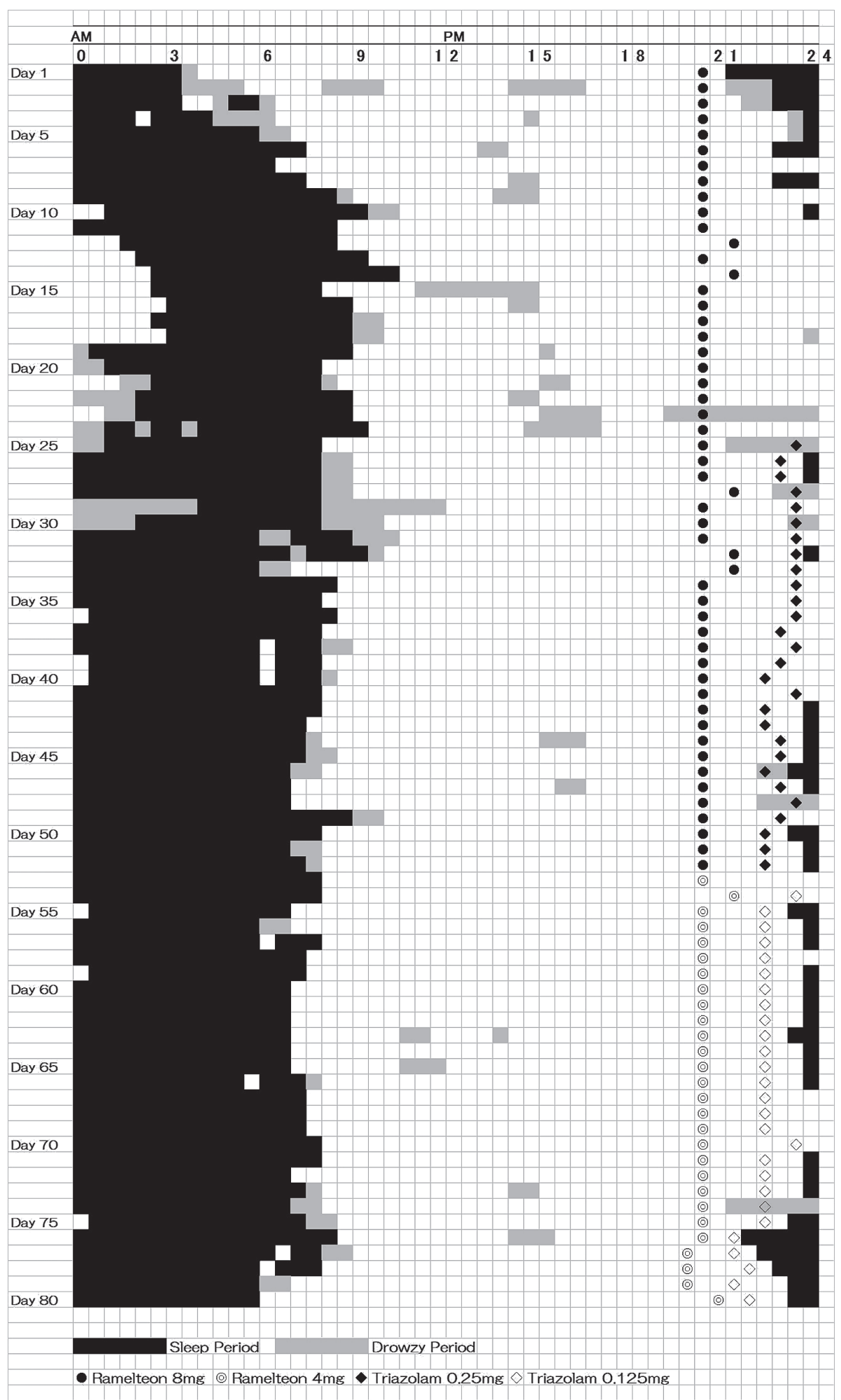

Fig. 1. Sleep diary of Patient 1.

Before starting of the treatment, both her sleep onset and offset times had gradually delayed by $1-3 \mathrm{~h}$ each day for nearly 4 month just the same as during 10 days from the start of the treatment. The treatment with ramelteon $8 \mathrm{mg} /$ day was initiated on day 1. Her sleep onset and offset times stopped free-running on day 15, but daytime sleepiness persisted. Triazolam $0.25 \mathrm{mg}$ /day at 23:00 was added from day 25 to promote earlier sleep onset. After starting medication of triazolam, her sleep latency was shortened, but difficulty awakening and morning sleepiness persisted. Sleep-wake schedule became to be normalized on day 34 with sleep onset at around 00:30 and awaking at around 08:00, and daytime sleepiness decreased. Doses of ramelteon and triazolam were reduced to $4 \mathrm{mg} / \mathrm{day}$ and $0.125 \mathrm{mg} / \mathrm{day}$, respectively, on day 53. Thereafter, the normalized sleep-wake schedule has been well kept. The detailed clinical course is shown in the text. 
From day 34, the sleep-wake schedule was improved with sleep onset at 00:30 and awaking at 08:00, and then morning sleepiness decreased. Her daily activity increased: she went out during the daytime more often (about 2 times per week) and began to do stretching exercises every day. At that time, depressive symptoms, such as loss of motivation and low self-esteem, had also improved, and she was keen to return to work. On day 53 after starting the treatment, the dose of ramelteon was reduced to a half tablet $(4 \mathrm{mg})$ and triazolam to $0.125 \mathrm{mg}$, and the sleep onset and awakening times remained unchanged. Three months after starting treatment, she began a part-time job as a nurse. After 1 year, she has stopped taking triazolam and no longer has sleep-onset insomnia. So far, she has maintained a stable sleep-wake schedule and her working activity with ramelteon treatment only.

\section{Patient 2}

A 33-year-old male presented with a chief complaint of prolonged sleep time and difficulty awakening at the proper time. There was no noteworthy physical disease or sight impairment. The patient had no previous sleep-related symptoms. After graduating from university, he began working in an international business company. From 2007, he usually worked in Chile and returned to Japan once every few months. In March 2010, after problems with staff at his workplace, he developed ideas of persecution, and took a leave of absence and returned to Japan. He visited a psychiatric clinic, where he was diagnosed as having a brief psychotic episode and prescribed olanzapine $5 \mathrm{mg}$ and risperidone $1 \mathrm{mg}$ per day, which resolved the ideas of persecution within a few days. However, shortly after starting recuperation at home in Japan in March 2010, he began to have difficulty with sleeping and awakening at a desirable time to permit being with his family; his sleep-wake schedule started to gradually delay by $2 \mathrm{~h}$ each day. In addition, the sleep duration lengthened. In December 2010, he was referred to us by his attending physician because his sleep pattern seemed to prevent him from returning to work and he felt depressed.

On presentation, he stated that both his sleep onset and offset times had continued to gradually delay by $2 \mathrm{~h}$ each day, which looked like a schedule with a $26-\mathrm{h}$ period, while simultaneously his average sleep duration had increased to $14 \mathrm{~h}$ per day. This condition had persisted for nearly 8 months. He was diagnosed as having FRT based on the clinical interview and his sleep diary, and treatment with ramelteon $8 \mathrm{mg}$ at 20:00 and methylcobalamin (a vitamin B12 preparation) $500 \mu \mathrm{g}$ after each meal $(1,500 \mu \mathrm{g} /$ day $)$ was begun. His sleep onset time before treatment was 23:00. The patient was instructed to avoid light exposure from $1 \mathrm{~h}$ prior to sleep onset, and to expose himself to sunlight in the morning hours after awakening. Free-running stopped immediately after starting the treatment, and his sleep onset time became regular at 22:00 to 23:00, but his sleep offset time was unstable, occurring between 08:00 and 11:00. Mean nighttime sleep duration at that time was 10-12 h long. About 3 weeks later, his mood was improved and sleep duration had shortened to about $8 \mathrm{~h}$, with the awakening time moving forward to between 06:00 and 07:00, while sleep onset time remained the same. One month later, his sleep onset time became fixed at 21:30 and the awakening time at 06:30, and he could return to work in Japan. One year later, he stopped taking both ramelteon and methylcobalamin and returned to a new post in South America for 3 months, without disturbance of the sleepwake rhythm. However, one month after returning to Japan for a holiday from South America, the previous free-running sleep-wake period recurred. He resumed taking ramelteon at 20:00 and methylcobalamin after each meal, and his sleep-wake rhythm improved within a few days. $\mathrm{He}$ is currently working in South America as before, and he remains on a good sleep-wake schedule without ramelteon or methylcobalamin treatment.

\section{Discussion}

Both of the current patients achieved a favorable sleepwake schedule with the melatonin agonist treatment and were able to return to normal social functioning. Patient 1 had a tendency for DSPT from her childhood, and had just managed to function in society in spite of it, but after leaving regular work developed FRT, presumably facilitated by a loss of social zeitgebers (Okawa and Uchiyama 2007). Patient 2 experienced episodes of FRT after returning to Japan from abroad. Although we cannot rule out the possibility that the 13-h time difference between South America and Japan was contributory, the loss of social zeitgebers after returning to Japan seems to have been the main factor for the onset of FRT, since he did not relapse after returning to work in South America. Given this history, it is possible that the symptoms may recur if he returns to Japan again for a long vacation.

We speculate that in our two sighted cases, the loss of social zeitgebers and presence of factors predisposing to CRSD, such as an impaired response of the circadian clock, seem to be risk factors for development of FRT, rather than inappropriate exposure to light.

For Patient 1, the relationship between the past depressive episodes and the CRSD is unclear. However, her depressive symptoms, which were clearly observed at the first visit, gradually resolved along with the improvement of the sleep-wake rhythm after starting treatment for FRT, suggesting that her depressive symptoms were secondary to the FRT. In Patient 2, psychotic symptoms were not observed at the first visit to our clinic and did not reappear during the second episode of FRT, suggesting that his psychotic symptoms preceding the first FRT onset were not related to the FRT.

Melatonin binding at the MT1 receptor exerts a sleepinducing effect by suppressing the neural activity for maintaining wakefulness (Reppert et al. 1994; Pandi-Perumal et al. 2007), whereas binding at the MT2 receptor results in a 
phase-shift of the circadian rhythm (Reppert et al. 1995). Ramelteon acts as a selective agonist for the MT1 and MT2 receptors; it has a time to maximum blood concentration of $0.75 \mathrm{~h}$ and a half-life of $1.14 \pm 0.39 \mathrm{~h}$. In Patient 1 , the action at the MT2 receptor was thought to play a major role in the effectiveness of ramelteon, because possible hypnotic action mediated by the MT1 receptor did not appear even during the periods when the blood concentration was probably high. On the other hand, in Patient 2, MT1 activity might have contributed to the therapeutic effect in addition to MT2 effects, since he was able to fall asleep within 2-3 h after taking the drug beginning on the first day of treatment.

According to previous reports, when taking melatonin for the treatment of FRT, an effective approach is to take 0.5-3 mg in several divided doses or a single dose a few hours before the desired sleep onset time (from evening to around 20:00) and to start the treatment when progressively delayed sleep onset time is near to the time desired (McArthur et al. 1996; Hayakawa et al. 1998; Okawa et al. 1998a, b; Sack et al. 2000). The timing of ramelteon treatment in the two cases reported here was based on previous reports of melatonin treatment. Free running stopped on the first day of treatment in Patient 2 and on day 15 of treatment in Patient 1. This response time difference between the two patients was possibly due to individual differences, as suggested by results of previous studies demonstrating that the period from the start of melatonin treatment until suppression of free-running varies considerably, from a few days to about 40 days (McArthur et al. 1996; Sack et al. 2000).

Melatonin has a half-life of about $0.5 \mathrm{~h}$, while ramelteon has a longer half-life, as indicated above. Considering the phase-response curve (Lewy et al. 1998), this longer half-life might prove more useful for targeting the drug action timing to provide a sufficiently therapeutic effect for FRT. On the other hand, it has been reported that, among healthy adults, ramelteon $(1 \mathrm{mg}, 2 \mathrm{mg}$, or $4 \mathrm{mg}$ ) administration before bedtime significantly advanced the circadian rhythm phase after a forced 5-h advance in the sleep-wake schedule, and that with an $8 \mathrm{mg}$ dose the change in dimlight melatonin offset (DLMoff) as the index of circadian rhythm was not significantly different from that of the placebo treated condition (Richardson et al. 2008). Based on this report, for Patient 1, the dose of ramelteon was reduced from $8 \mathrm{mg}$ /day to $4 \mathrm{mg}$ /day on treatment day 53 . Considering the 3-fold higher affinity of ramelteon for the MT2 receptor, compared to melatonin, a smaller dose of ramelteon may be appropriate to treat FRT.

Our patients took triazolam (Patient 1) and vitamin B12 (Patient 2) in combination with ramelteon, and were also given sleep hygiene education. It is possible that these interventions supportively acted to stabilize the circadian rhythm phase (Barion and Zee 2007; Dodson and Zee 2010). In Patient 1, the additional treatment with triazolam produced a sleep-inducing effect, but did not lead to an advanced waking time. The sleep-wake schedule was also unchanged after cessation of triazolam. Taking these facts into consideration, triazolam was therefore unlikely to have had a therapeutic effect for the FRT. On the other hand, vitamin $\mathrm{B} 12$ has been reported to promote entrainment by increasing biological clock photosensitivity (Tsujimaru et al. 1992). Thus, in Patient 2, vitamin B12 and ramelteon may have acted additively or synergistically to entrain the circadian clock.

This case report includes the following limitations. Because of the loss of the sleep diaries and the lack of actigraphic measurements, the diagnosis for those two patients was probable FRT, according to ICSD-2 criteria. In addition, neither dim-light melatonin onset nor DLMoff were performed, and levels of daily urinary 6-sulfatoxy-melatonin or other chronobiological markers were not ascertained in order to examine the cause of FRT and to determine the proper timing to initiate ramelteon therapy.

In conclusion, the course of these two patients has suggested that the melatonin agonist treatment is potentially useful for FRT, and that the combined use of sleep hygiene education and co-administration of vitamin B12 may enhance its treatment effectiveness.

\section{Conflict of Interest}

The authors declare no conflict of interest.

\section{References}

American Academy of Sleep Medicine (2005) The International Classification of Sleep Disorders, Diagnostic \& Coding Manual, 2nd ed., American Academy of Sleep Medicine, Westchester, IL, pp. 117-136.

American Academy of Sleep Medicine (2014) The International Classification of Sleep Disorders, Diagnostic \& Coding Manual, 3rd ed., American Academy of Sleep Medicine, Darien, IL, pp. 189-224.

Aoki, H., Ozeki, Y. \& Yamada, N. (2001) Hypersensitivity of melatonin suppression in response to light in patients with delayed sleep phase syndrome. Chronobiol. Int., 18, 263-271.

Barion, A. \& Zee, P.C. (2007) A clinical approach to circadian rhythm sleep disorders. Sleep Med., 8, 566-577.

Dodson, E.R. \& Zee, P.C. (2010) Therapeutics for Circadian Rhythm Sleep Disorders. Sleep Med. Clin., 5, 701-715.

Hashimoto, S., Nakamura, K., Honma, S. \& Honma, K. (1997) Free-running circadian rhythm of melatonin in a sighted man despite a 24-hour sleep pattern: a non-24-hour circadian syndrome. Psychiatry Clin. Neurosci., 51, 109-114.

Hayakawa, T., Kamei, Y., Urata, J., Shibui, K., Ozaki, S., Uchiyama, M. \& Okawa, M. (1998) Trials of bright light exposure and melatonin administration in a patient with non-24 hour sleep-wake syndrome. Psychiatry Clin. Neurosci., 52, 261-262.

Hayakawa, T., Uchiyama, M., Kamei, Y., Shibui, K., Tagaya, H., Asada, T., Okawa, M., Urata, J. \& Takahashi, K. (2005) Clinical analyses of sighted patients with non-24-hour sleep-wake syndrome: a study of 57 consecutively diagnosed cases. Sleep, 28, 945-952.

Hoban, T.M., Sack, R.L., Lewy, A.J., Miller, L.S. \& Singer, C.M. (1989) Entrainment of a free-running human with bright light? Chronobiol. Int., 6, 347-353.

Honma, K., Hashimoto, S., Endo, T. \& Honma, S. (1997) Light and plasma melatonin rhythm in humans. Biol. Signals, 6, 307-312. 
Karim, A., Tolbert, D. \& Cao, C. (2006) Disposition kinetics and tolerance of escalating single doses of ramelteon, a highaffinity MT1 and MT2 melatonin receptor agonist indicated for treatment of insomnia. J. Clin. Pharmacol., 46,140-148.

Kato, K., Hirai, K., Nishiyama, K., Uchikawa, O., Fukatsu, K., Ohkawa, S., Kawamata, Y., Hinuma, S. \& Miyamoto, M. (2005) Neurochemical properties of ramelteon (TAK-375), a selective MT1/MT2 receptor agonist. Neuropharmacology, 48, 301-310.

Lewy, A.J., Bauer, V.K., Ahmed, S., Thomas, K.H., Cutler, N.L., Singer, C.M., Moffit, M.T. \& Sack, R.L. (1998) The human phase response curve (PRC) to melatonin is about 12 hours out of phase with the PRC to light. Chronobiol. Int., 15, 71-83.

McArthur, A.J., Lewy, A.J. \& Sack, R.L. (1996) Non-24-hour sleep-wake syndrome in a sighted man: circadian rhythm studies and efficacy of melatonin treatment. Sleep, 19, 544-553.

Morgenthaler, T.I., Lee-Chiong, T., Alessi, C., Friedman, L., Aurora, R.N., Boehlecke, B., Brown, T., Chesson, A.L. Jr., Kapur, V., Maganti, R., Owens, J., Pancer, J., Swick, T.J. \& Zak, R.; Standards of Practice Committee of the American Academy of Sleep Medicine (2007) Practice parameters for the clinical evaluation and treatment of circadian rhythm sleep disorders. An American Academy of Sleep Medicine report. Sleep, 30, 1445-1459.

Ohta, T., Ando, K., Iwata, T., Ozaki, N., Kayukawa, Y., Terashima, M., Okada, T. \& Kasahara, Y. (1991) Treatment of persistent sleep-wake schedule disorders in adolescents with methylcobalamin (vitamin B12). Sleep, 14, 414-418.

Okawa, M., Mishima, K., Nanami, T., Shimizu, T., Iijima, S., Hishikawa, Y. \& Takahashi, K. (1990) Vitamin B12 treatment for sleep-wake rhythm disorders. Sleep, 13, 15-23.

Okawa, M. \& Uchiyama, M. (2007) Circadian rhythm sleep disorders: characteristics and entrainment pathology in delayed sleep phase and non-24-h sleep-wake syndrome. Sleep Med. Rev., 11, 485-496.

Okawa, M., Uchiyama, M., Ozaki, S., Shibui, K. \& Ichikawa, H. (1998a) Circadian rhythm sleep disorders in adolescents: clinical trials of combined treatments based on chronobiology. Psychiatry Clin. Neurosci., 52, 483-490.

Okawa, M., Uchiyama, M., Ozaki, S., Shibui, K., Kamei, Y., Hayakawa, T. \& Urata, J. (1998b) Melatonin treatment for circadian rhythm sleep disorders. Psychiatry Clin. Neurosci., 52, 259-260.

Oren, D.A., Giesen, H.A. \& Wehr, T.A. (1997) Restoration of detectable melatonin after entrainment to a 24-hour schedule in a 'free-running' man. Psychoneuroendocrinology, 22, 39-52.

Pandi-Perumal, S.R., Srinivasan, V., Poeggeler, B., Hardeland, R. \& Cardinali, D.P. (2007) Drug Insight: the use of melatonergic agonists for the treatment of insomnia-focus on ramelteon. Nat. Clin. Pract. Neurol., 3, 221-228.

Reppert, S.M., Godson, C., Mahle, C.D., Weaver, D.R., Slaugenhaupt, S.A. \& Gusella, J.F. (1995) Molecular characterization of a second melatonin receptor expressed in human retina and brain: the Mel1b melatonin receptor. Proc. Natl. Acad. Sci. USA, 92, 8734-8738.

Reppert, S.M., Weaver, D.R. \& Ebisawa, T. (1994) Cloning and characterization of a mammalian melatonin receptor that mediates reproductive and circadian responses. Neuron, 13, $1177-1185$

Richardson, G.S., Zee, P.C., Wang-Weigand, S., Rodriguez, L. \& Peng, X. (2008) Circadian phase-shifting effects of repeated ramelteon administration in healthy adults. J. Clin. Sleep Med., 4, 456-461.

Sack, R.L., Brandes, R.W., Kendall, A.R. \& Lewy, A.J. (2000) Entrainment of free-running circadian rhythms by melatonin in blind people. N. Engl. J. Med., 343, 1070-1077.

Tsujimaru, S., Ida, Y., Satoh, H., Egami, H., Shirao, I., Mukasa, H. \& Nakazawa, Y. (1992) Vitamin B12 accelerates re-entrainment of activity rhythms in rats. Life Sci., 50, 1843-1850.

Watanabe, T., Kajimura, N., Kato, M., Sekimoto, M., Hori, T. \& Takahashi, K. (2000) Case of a non-24 h sleep-wake syndrome patient improved by phototherapy. Psychiatry Clin. Neurosci., 54, 369-370. 